CC motif ligand 2 chemokine (CCL2) were measured in PMR patients and in healthy controls.

Results: A total of 25 patients with PMR were studied (32\% men; $68 \%$ women). The mean age was $72.72 \pm 9.32$ years (range 65-79). We also included 35 healthy controls. In reference to laboratory parameters and proinflammatory biomarkers at the onset of PMR, the mean values ( \pm standard deviation) were as follows: C reactive protein (CRP): $28.4 \pm 26.6 \mathrm{mg} / \mathrm{dL}$; erythrocyte sedimentation rate (ESR): $54.3 \pm 21.3 \mathrm{~mm} / \mathrm{h}$; haemoglobin: $12.6 \pm 1.6 \mathrm{~g} / \mathrm{dL}$; IL-6: $17.6 \pm 30.1 \mathrm{pg} / \mathrm{mL}$; IL-8: $10.1 \pm 6.9 \mathrm{pg} / \mathrm{mL}$; CXCL10: $259.4 \pm 290.7 \mathrm{pg} / \mathrm{mL}$; CXCL9:5175 $\pm 4396.8 \mathrm{pg} /$ $\mathrm{mL} ;$, CXCL2: $25.9 \pm 12.5 \mathrm{pg} / \mathrm{mL}$; CCL2: $471.3 \pm 152.9 \mathrm{pg} / \mathrm{mL}$.

IL-6, IL-8 and CXCL9 levels were significantly increased in PMR patients comparing with the healthy control group.

The Health Assessment Questionnaire (HAQ) performed at the onset of the PMR yielded a mean value of $1.6 \pm 0.6$. The mean dose of prednisone employed at the onset of PMR was $14.8 \pm 5.6 \mathrm{mg} /$ day.

After 6 months, the mean dose of prednisone employed was $6.7 \pm 3.9 \mathrm{mg} / \mathrm{day}$, the median [range] value for CRP was 3.1 [1.75-7.2] $\mathrm{mg} / \mathrm{dl}$ and the mean value for ESR was $22 \pm 14 \mathrm{~mm} / \mathrm{h}$. Regarding relationship between proinflammatory biomarkers studied and doses of prednisone employed, no differences were observed.

Regarding pain perception and disability, we observed higher visual analogic scale values (VAS) in association with higher IL-6 levels $(P=0.03)$ and higher $\mathrm{HAQ}$ values in association with higher IL-6 and CXCL2 values.

After 6 months, our patients showed an excellent evolution, and no complications were observed.

Conclusion: During the acute onset of PMR, IL-6, IL-8 and CXCL9 levels were significantly increased in our population of PMR patients. No differences were observed between proinflammatory biomarkers studied and doses of prednisone employed.

Disclosure of Interests: None declared

DOI: 10.1136/annrheumdis-2021-eular.3114

\section{POS1366 THE RELATIONSHIP OF MAST CELL ACTIVATION SYNDROME AND HYPERMOBILE EHLERS-DANLOS SYNDROME IN HOSPITALIZED PATIENTS IN THE UNITED STATES}

K. Mathias ${ }^{1}$, A. Mantha ${ }^{2}$, L. Mathias ${ }^{3}$, D. Arkfeld ${ }^{3}{ }^{1}$ University of Chicago, Internal Medicine, Chicago, United States of America; ${ }^{2}$ USC Keck School of Medicine, Internal Medicine, Los Angeles, United States of America; ${ }^{3}$ USC Keck School of Medicine, Rheumatology, Los Angeles, United States of America

Background: Patients with hypermobile Ehlers-Danlos syndrome (hEDS) often report symptoms of cutaneous flushing, pruritis, vomiting, diarrhea and anaphylactoid responses to environmental, food, and mechanical stimuli. There is a growing body of literature investigating the possible link between disorders of mast cell regulation including mast cell activation syndrome (MCAS) and connective tissue disorders including hEDS. The evidence for such a relationship, however, remains limited ${ }^{1}$.

Objectives: We aimed to evaluate the association between hEDS and MCAS among hospitalized patients using a nationally representative cohort.

Methods: Hospitalized patients with a diagnosis of hEDS or MCAS were identified in the 2016-2018 National Inpatient Sample (NIS) using the International Classification of Diseases 10 system (ICD-10). The NIS is an all-payer inpatient database that estimates over 37 million annual U.S. hospitalizations and is maintained by the Healthcare Cost and Utilization Project. The primary outcomes were prevalence of EDS and MCAS among hospitalized patients. Secondary outcomes included cause of admission, mortality, length of stay, and cost of care. Multivariate hierarchical regression analysis was using adjusting for demographics, hospital factors, and comorbid conditions.

Results: Among 37,665 patients identified in this study, 35,115 (95.9\%) patients had hEDS and 3,630 (9.6\%) patients had MCAS. 1080 patients had concomitant diagnosis of hEDS (3.1\%) and MCAS (29.8\%), of whom $555(51.4 \%)$ were diagnosed in 2018 compared with $100(9.3 \%)$ patients in $2016(\mathrm{P}<0.001)$. The mean age of patients with both conditions was significantly lower than those diagnosed with hEDS (29.5 vs 36.6 years, $\mathrm{P}<0.001)$ or MCAS (29.5 vs 39.8 years, $\mathrm{P}<0.001$ ) alone. Those with comorbid hEDS and MCAS were more likely female (95.8\% vs $84.5 \%, \mathrm{P}<0.001)$, had private insurance $(67.6 \%$ vs $52.4 \%, \mathrm{P}<0.001)$ and were among the highest income quartile (47.4\% vs $31.9 \%, \mathrm{P}<0.001)$. There were no significant differences in mortality or overall comorbidity burden. Patients with hEDS and MCAS had higher adjusted hospitalization charges $(\$ 64,445$ vs $\$ 54,782, \beta=0.15, P<0.05)$ and longer adjusted lengths of stay (6.4 vs 4.9 days, IRR 1.35, $\mathrm{P}<0.001$ ).

Conclusion: Nearly 1 in 3 patients diagnosed with MCAS have comorbid diagnosis of hEDS. This association appeared to increase over time with the increasing diagnosis of MCAS. Social determinants of disease such as health insurance status and income appeared to be associated with the identification of the overlap syndrome. These findings merit further investigation into the co-occurrence of these disease entities and development of consistent clinical diagnostic criteria. REFERENCES:

[1] Kohn A, Chang C. The Relationship Between Hypermobile Ehlers-Danlos Syndrome (hEDS), Postural Orthostatic Tachycardia Syndrome (POTS) and Mast Cell Activation Syndrome (MCAS). Clin Rev Allergy Immunol. 2020;58(3):273-297. doi:10.1007/s12016-019-08755-8

Disclosure of Interests: None declared

DOI: 10.1136/annrheumdis-2021-eular.3177

\section{POS1367 PROCALCITONIN IN RHEUMATOLOGY}

K. Erraoui ${ }^{1}$, K. Nassar ${ }^{2}$, S. Janani ${ }^{1} .{ }^{1} \mathrm{CHU}$ Ibn Rochd, Department of Rheumatology, Casablanca, Morocco; ${ }^{2} \mathrm{CHU} \mathrm{Ibn} \mathrm{Rochd,} \mathrm{Department} \mathrm{of}$ Rheumatology, Casablanca, Morocco

Background: The differentiation between infection and aseptic inflammation is difficult and often a challenge in the daily practice of rheumatology.Procalcitonin (PCT) is a preferred marker for bacterial infections that allows the rheumatologist to make an early diagnosis, choose an appropriate antibiotic therapy for bacterial infection, and avoid unnecessary antibiotic therapy for inflammation.

Objectives: To study the levels of PCT in various inflammatory states observed in a rheumatology department, and to assess its possible discriminating role in the differentiation of bacterial infection from other inflammatory processes.

Methods: This is a preliminary monocentric retrospective study conducted from January 2018 to December 2020 in our rheumatology department. The inclusion criteria were all patients hospitalized in rheumatology for a rheumatic or autoimmune disease or a bone and joint infection.PCT samples from patients were analyzed along with general signs (fever/chills) and with routine assessment (blood count including white blood cell count(WBC), erythrocyte sedimentation rate (ESR)and $\mathrm{C}$ reactive protein(CRP)) in patients admitted to hospitalfor a fever or a biological inflammatory syndrome, or both, with a laboratory cut-off value of $0.5 \mathrm{ng} / \mathrm{ml}$.

Results: 20 patients were included. Their mean age was 46.1 (extremes 19-66) and the with sex ratio was 1 . Three patients had a history of autoimmune disease, 2 of neoplasia, 1 with chronic terminal kidney failure and hemodialysis, and 4 patients took antibiotics before hospitalization. The mean PCT was $100.01 \mathrm{ng}$ $\mathrm{ml}$ (extremes $0.01-200$ ), and Sensitivity at $57.44 \%$ and specificity at $100 \%$, PPV $100 \%$, NPV: $81.25 \%$.

The patients were divided according to their final diagnosis into 3 groups: bacterial or fungal infection; inflammatory abacterian disease (autoimmune or rheumatic flare, neoplasia, metabolic, reactive arthritis); infection + inflammatory abacterian disease. Group 1 included 4 patients, their mean PCT was $6.92 \mathrm{ng}$ $\mathrm{ml}(0.16-26.80)$, mean CRP was $131.725 \mathrm{mg} / \mathrm{L}$, and mean ESR was $50 \mathrm{~mm}$

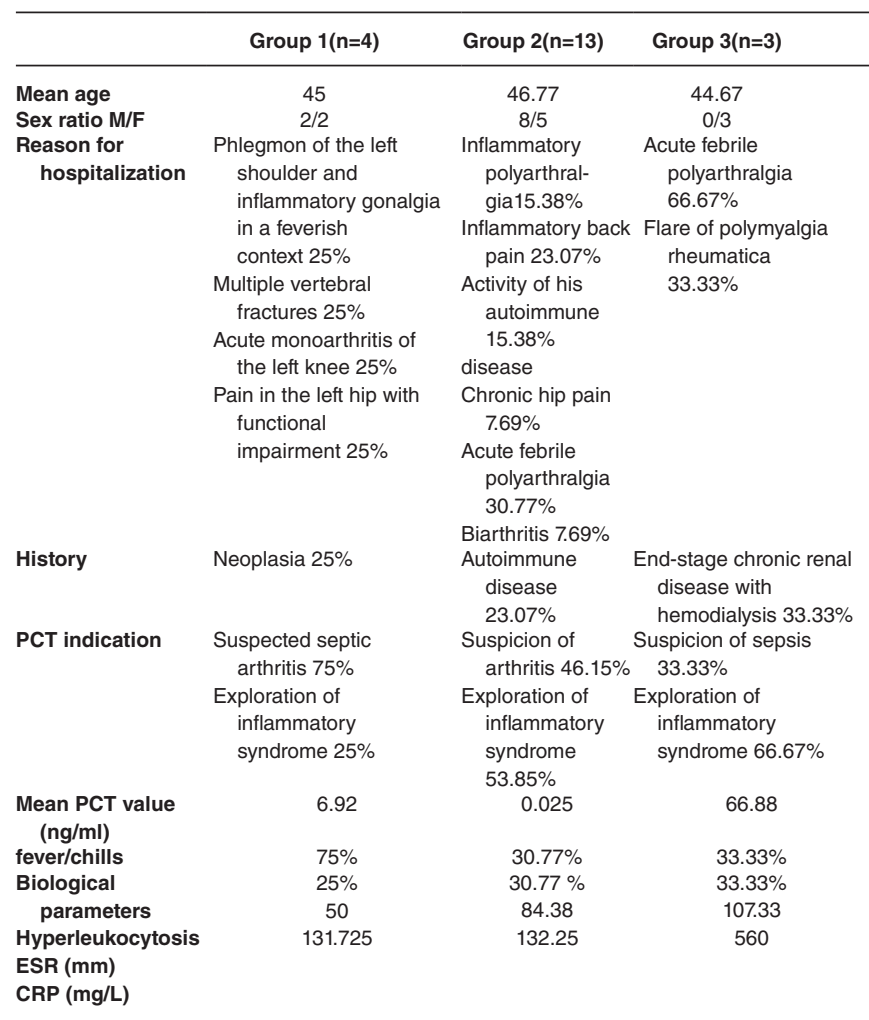

